\title{
Actividades físicas-recreativas e inclusión: Una experiencia con adultos parapléjicos de la Asociación Asopléjica
}

\author{
Physical activities - recreational and inclusion: An experience with \\ paraplegic adults from Asoplegic Association
}

Johanna Ladinez Garces. $^{1}$ \& Giceya de la Caridad Maqueira Caraballo. ${ }^{2}$

Recibido: 22-07-2020 / Revisado: 27-08-2020 /Aceptado: 25-09-2020/ Publicado: 06-11-2020

\begin{abstract}
.
DOI: https://doi.org/10.33262/cienciadigital.v4i4.1.1451

The care of people with physical-motor limitations (paraplegics), given the variability of cases, etiologies, forms of classification, level of commitment and performance that occurs between one paraplegic person and another, make the inclusion processes continue to constitute a great challenge. In an observation carried out in the Asoplegic Association of the city of Guayaquil, it was detected that regardless of the programs that are developed, there are limitations for the practice of physical-recreational activities by the paraplegic adults that make up the association, negatively affecting their performance and social inclusion. The research that is presented aims to: Provide a system of physical-recreational activities conducive to the inclusion of paraplegic adults from the Asoplegic Association of Guayaquil to the practice of physical sports activities. A descriptive, non-experimental methodology was followed with the use of theoretical and empirical methods and the use of techniques such as the survey, achieving the results of providing a system of physical-recreational activities, composed of 4 blocks (Gymnastic Activities in Wheelchairs, Activities Wheelchair Adapted Sports, Wheelchair Adapted Recreational Activities, Wheelchair Dance Therapy). The assessment made by the evaluating specialists who agreed on the usefulness and benefits of the proposed activity system was very significant, considering it as a timely alternative to facilitate the processes of inclusion of paraplegic adults who are members of the Asoplegic Association.
\end{abstract}

Keywords: Physical-recreational activities, adults, paraplegics, Asoplegic Association.

\footnotetext{
${ }^{1}$ Unidad Educativa José Joaquín Pino y Caza, johana.ladinez @educación.gob.ec

${ }^{2}$ Universidad de Guayaquil, Universidad Laica Vicente Rocafuerte y Universidad de las Fuerzas Armadas (ESPE), giceyamc@gmail.com.
} 


\section{Resumen:}

La atención a las personas con limitaciones físico-motrices (parapléjicos), dada la variabilidad de casos, etiologías, formas de clasificación, nivel de comprometimiento y desempeño que se da entre una persona parapléjica y otra, hacen que los procesos de inclusión sigan constituyendo un gran reto. En observación realizada en la Asociación Asopléjica de la ciudad de Guayaquil, se detectó que independientemente de los programas que se desarrollan, existen limitaciones para la práctica de actividades físicas-recreativas por parte de los adultos parapléjicos que integran la asociación, incidiendo negativamente en su desempeño e inclusión social. La investigación que se presenta tiene como objetivo: Aportar un sistema de actividades físicas -recreativas conducente a la inclusión de los adultos parapléjicos de la Asociación Asopléjica de Guayaquil a la práctica de actividades físicas deportivas. Se siguió una metodología descriptiva, no experimental con el empleo de métodos teóricos y empíricos y el uso de técnicas como la encuesta, lográndose como resultados aportar un sistema de actividades físicas-recreativas, compuesto por 4 bloques (Actividades Gimnásticas en Silla de Ruedas, Actividades Deportivas Adaptadas en Silla de Ruedas, Actividades Recreativas Adaptadas en Silla de Ruedas, Danza Terapia en Silla de Ruedas). Resulto muy significativo la valoración realizada por parte de los especialistas evaluadores quienes coinciden en la utilidad y beneficios del sistema de actividades propuesto, considerando este como una alternativa oportuna para facilitar los procesos de inclusión de los adultos parapléjicos integrantes de la Asociación Asopléjica.

Palabras clave: Actividades físicas-recreativas, adultos, parapléjicos, Asociación Asopléjica.

\section{Introducción.}

Datos ofrecidos por la Organización Mundial de la Salud, OMS, (2018), en el Informe Mundial sobre discapacidad señalan que más de 1000 millones de personas viven en el mundo con alguna forma de discapacidad, lo cual representa un $15 \%$ de la población mundial. Esto significa que entre 110 y 190 millones de personas presentan grandes dificultades para funcionar debido a la presencia de una o varias discapacidades.

En el caso de Ecuador según el Informe del Consejo Nacional para Discapacidades, (2017), existen registradas un total de 481.392 personas portadoras de algún tipo de discapacidad, resultando altamente alarmante la presencia de la discapacidad físicomotriz, la cual ocupa el 46,34 \% del total de personas con discapacidad registrada en el Registro Nacional de Discapacidad. Este mismo informe indica que 25,09 \% de esta población reside en la provincia de Guayas.

Puede apreciarse en las estadísticas y estudios realizados en los últimos años que debido a múltiples factores tanto a nivel internacional como nacional la presencia de personas 
con algún tipo de discapacidad se ha venido incrementado. Es por ello que buscar alternativas conducentes a perfeccionar la inclusión de este grupo poblacional que de por sí se encuentra entre los más afectados a nivel mundial sigue siendo una gran preocupación y ocupación.

Ecuador como país, ha venido realizando múltiples acciones encaminadas a posibilitar la optimización en los procesos de inclusión educativa y social de las personas que presentan algún tipo de discapacidad. Existe un marco legal y programático que respalda dichas acciones, garantizando el derecho a la salud, la educación, la atención integral y el desarrollo pleno de cada persona y de sus familias. Sin embargo aún no se logran alcanzar los niveles deseados y necesarios en cuanto a atención e inclusión de la población que presenta afectaciones físico-motrices.

En observación realizada en la Asociación Asopléjica de la ciudad de Guayaquil, se detectó que independientemente de los programas que se desarrollan, existen limitaciones para la práctica de actividades físicas-recreativas por parte de los adultos parapléjicos que integran la asociación, incidiendo negativamente en su desempeño e inclusión.

En consonancia con lo señalado la investigación que se presenta tiene como objetivo aportar un sistema de actividades físicas -recreativas conducente a la inclusión de los adultos parapléjicos de la Asociación Asopléjica de Guayaquil a la práctica de actividades físicas deportivas

\section{Generalidades de la discapacidad física -motriz y su relación con las actividades físicas -recreativas}

La discapacidad física - motriz debido a su propia naturaleza, variabilidad de casos, etiologías, formas de clasificación, nivel de comprometimiento y desempeño que se da entre una persona parapléjica y otra, hacen que los procesos de inclusión sigan constituyendo un gran reto.

Según: (Pizarro \& Veintimilla , 2016, pág. 31) la clasificación de las discapacidades físicas -motrices siguen los siguientes criterios:

\section{Tabla No 1. Clasificación según criterios}

Clasificación de las discapacidades Clasificación según la topografía de la motoras: afectación:

\begin{tabular}{ll|cl}
$\checkmark$ & Parálisis cerebral & $\checkmark$ & Hemiplejia \\
$\checkmark$ & Poliomielitis & $\checkmark$ & Diplejía \\
$\checkmark$ & Mielomeningocele o espina bífida & $\checkmark$ & Paraplejia \\
$\checkmark$ & Lesiones medulares & $\checkmark$ & Tetraplejia \\
$\checkmark$ & Distrofia muscular progresiva & $\checkmark$ & Monoplejia
\end{tabular}

Fuente: (Pizarro \& Veintimilla , 2016, pág. 31)

Se comparte con (Moreno \& Amaya , 2009) una idea esencial: para las personas con paraplejia, aprender a vivir en esta condición se convierte en un desafío permanente; 
precisamente porque las actividades que se consideraban "sencillas" como el baño, el vestirse, la eliminación urinaria e intestinal y los traslados, constituyen diariamente una prueba de resistencia física y psicológica, requiriendo la ayuda de otras personas, así como de adaptaciones ambientales y del uso de equipos especiales para realizarlas.

Lo anteriormente señalado se complejiza mucho más en los adultos parapléjicos, precisamente porque debido a la diversidad de formas y manifestaciones que presentan requieren de programas y actividades más específicas y adaptadas a su nivel de potencialidades, así como poder contar con personal capacitado y preparado para dar respuesta a la diversidad manifiesta en cada caso.

Se reconoce que las personas que manifiestan alguna discapacidad física, se enfrentan a grandes desafíos en la vida diaria que, a lo largo del camino son los propulsores en crear las potencialidades que se desarrollan a partir de una necesidad superior, en esto hace también referencia. (Rios , y otros, 2016, pág. 20) indicando que "Las motivaciones intrínsecas de la persona con discapacidad que practique Deporte o Actividad Física Adaptada van a potenciar estos beneficios físicos (sistemas cardiovascular, respiratorio, locomotor y metabólico-endocrino), sociológicos y psicológicos.

Estudios precedentes dentro de los cuales se destacan los aportes de Reina, 2003; Lanuna, Laiño \& Castro (2014), acompañados por Herrera, (2016), Pérez, (2017) y Ladinez, (2016), coinciden en aseverar la importancia de la práctica de actividades físicas recreativas en esta población. Insisten en los variados beneficios que las actividades físicas, deportivas y recreativas provocan a estas personas y comparten la idea de que es necesario crear todas las condiciones para poder desarrollar y ampliar las ofertas existentes.

Especial interés tiene la investigación realizada por (Tito, Ramiro, Rodriguez, Analuiza, \& Calero, 2016, pág. 14), los cuales demuestran la influencia de las actividades físicorecreativas en la autoestima del adulto mayor; los autores señalan que: “...el diseño de actividades físico-recreativas debe socializarse con los usuarios, incorporando los gustos y preferencias de estos, en pos de incrementar su motivación auto-determinada, y por ende la participación sistemática en los programas físico-recreativos”.

Según, Ladinez, (2016), "La paraplejia, no es un impedimento para que los adultos puedan realizar cualquier tipo de actividad física, está científicamente comprobado que la actividad física ayuda a mejorar el desarrollo morfo-funcional". Otras actividades como el folklor nacional en sus diferentes manifestaciones constituyen un medio de desarrollo biopsicosocial, que ayuda a elevar la autoestima no solo del adulto parapléjico si no de quienes lo practican" (pág 75)

En correspondencia con lo que se ha venido tratando se considera que la actividad física, de manera general influye directamente en el desarrollo físico y motor de quienes la practican, generando beneficios relacionados con su desempeño físico, psicológico y social. Su práctica sistemática repercute positivamente en la adaptación de las personas a su entorno, potencia el desarrollo de valores y aumenta la calidad de vida de quienes la 
practican; al prevenir la aparición de enfermedades acompañantes o compensar las limitaciones existentes productos de diferentes discapacidades.

En el caso de las personas parapléjicas ha quedado demostrado la efectividad de la práctica de actividades físicas recreativas ya que se contribuye a potenciar las partes conservadas, aumentan su validismo y desempeño posibilitando su inclusión educativa y social.

\section{Las actividades Físicas _ Recreativas y su interacción con la Inclusión educativa y social de las personas parapléjicas}

La inclusión educativa y social de las personas parapléjicas sigue constituyendo una gran desafío; máxime si tenemos en cuenta que dada su diversidad se requiere adaptar las diferentes estrategias de intervención y los materiales y recursos a utilizar.

Se coincide con Sailema A., Maqueira, Sailema, M., \& Sailema, AD (2019), en que en términos de inclusión y atención a la diversidad, es fundamental buscar alternativas didácticas y metodológicas basadas en los principios de normalización y contextualización, de modo tal que permitan adaptar lo general a lo particular en función del logro de los objetivos y habilidades propuestos.

Resulta evidente que al trabajar la inclusión y atención a la diversidad en el contexto de la práctica de actividades físicas, deportivas y recreativas tengamos presente la necesidad de adaptar tanto los recursos, como las formas de enseñanza, aprendizaje e incluso de evaluación del desempeño. (Castelo \& Maqueira, 2015).

En este sentido es importante no solo considerar que las personas con limitaciones físico -motriz tienen los mismos derechos para practicar actividades física deportivas y recreativas, sino también debemos tener en cuenta que dada su diversidad requieren de diferentes adaptaciones.

Apuntan (Barroso, Cordova, \& Calero, 2016), que: "El carácter inclusivo de la metodología a utilizar se expresa al asegurar el derecho de las personas con limitaciones físico-motoras a la práctica de actividades físico-deportivas, con independencia del tipo de discapacidad predominante, o el grado de la misma, atendiendo a la individualidad desde la diversidad y utilizando recursos adicionales o la adaptación de los disponibles de acuerdo a los requerimientos de los practicantes".

Lo expuesto coincide íntegramente con el propósito del estudio que se presenta ya que se parte del principio del derecho a la inclusión de las personas con limitaciones físicomotriz a la práctica de actividades físicas, deportivas y recreativas.

Se considera que además de ser un derecho se precisa de adaptar las formas, estrategias, metodologías, recursos, evaluaciones con el fin de poder mediante la práctica física deportiva y recreativa posibilitar sus procesos de inclusión. Especial interés tiene poder contar con actividades diseñadas y adaptadas a las potencialidades de los adultos parapléjicos. 


\section{Metodología}

Se empleó un diseño investigativo con enfoque mixto mediante la articulación de paradigmas cualitativos y cuantitativos, con énfasis en la investigación de tipo descriptiva, de campo no experimental y la utilización de métodos teóricos y empíricos, los cuales unidos a las técnicas como la encuesta y la entrevista permitieron obtener resultados muy valiosos.

La investigación fue desarrollada en la Asociación Asopléjica, de la ciudad de Guayaquil. En esta asociación se encuentran afiliados niños, niñas, jóvenes, adultos y adultos mayores con diversas discapacidades como la física, intelectual y sensorial, de los cuales 22 son adultos con paraplejia que representan la población y muestra de la investigación realizada.

Tabla No 2. Población y Muestra

\begin{tabular}{lcc}
\hline Rango de edad & Hombres & Mujeres \\
\hline $25-30$ & 1 & 0 \\
\hline $30-40$ & 2 & 6 \\
\hline $40-50$ & 3 & 8 \\
\hline $50-60$ & 1 & 1 \\
\hline Total & 7 & 15 \\
\hline
\end{tabular}

Elaborado por: Ladinez, Johanna (2020)

Dentro de los criterios de inclusión determinados para la conformación de la muestra se encuentran:
$\checkmark$ Presentar una discapacidad motriz clasificada como paraplejia
$\checkmark$ Tener motivación para participar en la investigación
$\checkmark$ Tener una condición física favorable

\section{Análisis y Discusión de Resultados}

Después de la aplicación de los instrumentos y técnicas seleccionadas, se aprecia que en la Asociación Asopléjica, de la ciudad de Guayaquil, existe un déficit de personal especializado y capacitado para desarrollar la práctica actividades físicas - recreativas, con este tipo de población, lo cual genera sedentarismo y escasas oportunidades para mejorar su calidad de vida, de la población adulta con parapléjia, causando así la exclusión a diversos eventos deportivos, culturales artísticos entre otros. Todo lo anterior permite confirmar aspectos de gran interés en el proceso investigativo desarrollado:

Quedó confirmada la importancia, pertinencia y actualidad del tema de investigación, así como la necesidad de buscar soluciones que permitan perfeccionar los procesos de 
inclusión de las personas adultas parapléjicas a las actividades físicas recreativas. Esto nos hace coincidir con los estudios precedentes realizados por autores como: Castelo \& Maqueira, 2015, Barroso, Cordova, \& Calero, 2016, Ladinez, (2016) y Sailema A., Maqueira, Sailema, M., \& Sailema, AD (2019), los cuales exponen los beneficios de las actividades físicas-deportivas para la inclusión de estas personas.

- Se logra demostrar que existen limitaciones en cuanto al déficit de personal especializado y capacitado que puedan desarrollar y poner en práctica metodologías inclusivas mediante la práctica de actividades físicas - recreativas para este tipo de población.

- Resultó muy significativo la receptividad, interés y motivación mostrada por las personas parapléjicas participantes en cuanto a la aceptación de la práctica de actividades físicas deportivas y recreativas.

- Se confirma la necesidad de diseñar un Sistema de actividades físicas-recreativas para personas adultas con paraplejía como opción viable para desarrollar los procesos de inclusión

Lo destacado provoco la necesidad de diseñar un Sistema de Actividades físicarecreativas conducentes a perfeccionar los procesos de inclusión de los adultos parapléjicos a la práctica de actividades físicas deportivas. Para su diseño se utilizó el método Sistémico- Estructural Funcional, el cual permitió modelar la propuesta, quedando conformada en 4 bloques: Bloque 1: Actividades Gimnásticas en Silla de Ruedas, Bloque2: Actividades Deportivas Adaptadas en Silla de Ruedas, Bloque 3: Actividades Recreativas Adaptadas en Silla de Ruedas, Bloque 4: Danza Terapia en Silla de Ruedas.

\section{Ejemplo de actividades propuesta para cada bloque:}

Bloque $\mathbf{N}^{\circ} 1$ Actividades Gimnásticas adaptadas en silla de ruedas

Objetivo: Profundizar en el aumento de la capacidad motora de manera integral para la estimulación de nuevas habilidades.

\section{Descripción:}

1. Gimnasia con bastones

2. Gimnasia con pelota flexible de mano

3. Gimnasia con mancuernas

4. Gimnasia con banda elástica

5. Gimnasia con hula hula

\section{Recursos:}

Bastones de madera de $1.30 \mathrm{mts}$

$\checkmark$ Pelotas flexibles con pupitos de $22 \mathrm{~cm}$ de diámetro

$\checkmark$ Mancuernas de 1,2 y $4 \mathrm{~kg}$

$\checkmark$ Banda elástica suave y médium

$\checkmark$ Hula hula de $120 \mathrm{~cm}$ de diámetro 
Bloque $\mathbf{N}^{\circ} 2$

Actividades deportivas adaptadas en silla de ruedas

Objetivo: Potenciar las habilidades físicas específicas aumentando la autoestima e independencia en la participación de eventos deportivos

\section{Descripción:}

1. Para Baloncesto

2. Para bádminton

3. Para balonmano

4. Para Tenis de campo

\section{Recursos:}

$\checkmark$ Balón de voleibol de $21 \mathrm{~cm}$ de diámetro

$\checkmark$ Balón de baloncesto $23 \mathrm{~cm}$ de diámetro

$\checkmark$ Volantes de nylon

$\checkmark$ Volantes de pluma de ganso

$\checkmark$ Balón de balonmano $12 \mathrm{~cm}$ de diámetro

$\checkmark$ Red de bádminton

$\checkmark$ Red de Voleibol

$\checkmark$ Red para tenis de campo

$\checkmark$ Pelota de tenis

$\checkmark$ Raquetas de tenis

$\checkmark$ Raquetas de bádminton

$\checkmark$ Arcos

\section{Bloque $\mathbf{N}^{\circ} \mathbf{3}$}

Actividades recreativas en silla de ruedasObjetivo: Fomentar el disfrute de las actividades recreativas al aire libre para fortalecer las habilidades sociales

\section{Descripción:}
$\checkmark$ Ajedrez
$\checkmark$ Yincanas

\section{Recursos:}
$\checkmark$ Tablero
$\checkmark$ Piezas
$\checkmark$ Parlante
$\checkmark$ Extensión
$\checkmark$ Pen drive
$\checkmark$ Accesorios

$\checkmark$ Presentaciones artísticas

$\checkmark$ Paseos recreativos 
Bloque $\mathbf{N}^{\circ} 4$ Danza terapia en silla de ruedas

Objetivo: Desarrollar habilidades expresivas y comunicativas

\section{Descripción:}

Bailo terapia en silla de ruedas

$\checkmark$ Folklore en silla de ruedas

\section{Recursos:}

$\checkmark$ Sillas de ruedas

$\checkmark$ Parlante

$\checkmark$ Pen drive

$\checkmark$ Accesorios

Es importante explicar que cada actividad se prevé realizarla en dos momentos: jornada matutina y nocturna, acomodando los horarios según posibilidades de los participantes y siguiendo la siguiente planificación:

Tabla No 3: Planificación de actividades semanales

\begin{tabular}{lllll}
\hline Lunes & Martes & Miércoles & Jueves & Viernes \\
\hline Actividades & Actividades & Danza terapia & Actividades & Viernes de amigos \\
Gimnásticas & Deportivas & & Recreativas & (Este día los \\
& & & $\begin{array}{l}\text { usuarios elegirán } \\
\end{array}$ \\
& & & $\begin{array}{l}\text { libremente las } \\
\text { actividades }\end{array}$ \\
& & & sociales de interés \\
& & & personal \\
& & & $(18 \mathrm{~h} 00$ a 21h00) \\
\hline
\end{tabular}

A continuación se expone un ejemplo de las fases consideradas para la aplicación de las actividades, la cual incluye la fase inicial, principal y final.

Tabla No 4: Ejemplo de las Fase de aplicación de las actividades

\section{FASE DE APLICACIÓN DE LAS ACTIVIDADES}

La preparación del cuerpo es primordial antes de realizar una

INICAL actividad física, deportiva o recreativa, para evitar futuras lesiones o descompensaciones, a esto le llamamos calentamiento, para lo cual realizaremos los siguientes ejercicios:

Recomendaciones: Se recomienda entre 8 a 10 repeticiones cada ejercicio.

Asegurar que la silla se encuentre en buenas condiciones antes de realizar la actividad 


\section{Movimientos de la cabeza}

- Recostar la cabeza del lado izquierdo y luego al lado derecho de una forma suave no acelerada.

- Llevar el mentón hacia el pecho y luego elevar el mentón de una forma suave no acelerada.

\section{Movimiento de hombros:}

- Elevar los dos hombros al mismo tiempo y lo llevamos hacia atrás, luego hacia adelante.

- Subir y bajar los hombros alternadamente

\section{Movimiento de brazos:}

- Extender los brazos a la altura del pecho, luego bajarlos de manera alternada

- Extender los brazos a la altura del pecho y luego abrirlos de forma horizontal de manera alternada.

- Extender los brazos y luego contraer de manera alternada

- Elevar los brazos por encima de la cabeza y luego bajarlos de manera alternada

- Elevar los brazos a la altura de la cabeza y bajarlos hasta quedar en posición horizontal, hacerlo de manera alternada

- Alternación de brazos simulando una caminata

- Rotación de muñeca izquierda y luego derecha

- Abrir y cerrar las falanges

\section{Flexión de tronco}

- Colocar las manos en la cintura y flexionar el tronco hacia la derecha, regresamos al centro y luego a la izquierda.

- Con brazos en la cintura flexionamos el tronco hacia adelante y regresamos a la posición inicial

Esta fase está enfocada en la aplicación de la actividad planificada, utilizando todos los medios y recursos disponibles, los temas seleccionados son:

- Gimnasia con bastones

- Gimnasia con pelota de mano flexible

- Gimnasia con mancuernas

- Gimnasia con banda elástica

- Gimnasia con hula hula

- ParaBaloncesto

- Parabádminton

- Para balonmano

- Para tenis de campo

- Ajedrez 
- Presentaciones artísticas

- Paseos recreativos

- Yincanas

- Bailo terapia en silla de ruedas

- Folklore en silla de ruedas

FINAL

En esta fase se debe bajar la intensidad, con actividades específicas, a esto le llamamos vuelta a la calma, para lo cual realizaremos los siguientes ejercicios:

\section{Estiramiento del músculo del cuello}

- Poner la mano derecha en la oreja izquierda y estirar el músculo hacia el mismo lado derecho, repetirlo con el lado izquierdo

\section{Estiramiento de los músculos del pecho}

- Llevar los brazos al mismo tiempo hacia atrás de la silla y elevar el mentón

Estiramiento de los músculos del hombro, brazos y espalda

- Colocar la mano derecha al nivel del hombro izquierdo y con la mano izquierda hacer una leve presión en el codo del brazo derecho llevándolo hacia arriba, hasta que los dedos de la mano derecha alcancen el centro de la espalda, luego hacerlo del lado contrario.

- Cruzar los brazos de hombro a hombro y con las yemas de los dedos tratar de tocar la espalda

- Entrelazar los dedos y elevar los brazos lo más que puedan

- Poner la mano derecha en la cintura y elevar el brazo contrario inclinándose hacia el mismo lado, repetir el ejercicio con el otro lado.

- Poner las manos al nivel de las lumbares elevamos el pecho y el mentón hacia arriba

- Agarrándose con una mano de la silla, nos inclinamos hacia adelante estirando el otro brazo, repetimos el ejercicio con el otro brazo.

Elaborado por: Ladinez, (2020)

Como puede apreciarse la propuesta está concebida según las posibilidades de los participantes, la misma prevé la adaptabilidad tanto de las estrategias a seguir como la variabilidad y adaptabilidad de los recursos, materiales, formas de participación, evaluación y niveles de desempeño. Todo ello garantiza su funcionabilidad, pertinencia y utilidad. 


\section{Conclusiones}

- Se confirma la importancia y beneficios de la práctica de actividades físicas recreativas para mejorar la condición física y los procesos de inclusión de las personas con paraplejia que integran la asociación Asopléjica de la ciudad de Guayaquil. Estas actividades inciden positivamente en su autoestima, previenen enfermedades y ayudan a la compensación de las lesiones existentes al tener esa condición.

- El diagnóstico realizado evidenció la necesidad de encontrar alternativas que contribuyan a poner en práctica el sistema de actividades físicos- recreativas propuesto para contribuir a la solución de la problemática motivo de estudio.

- Se logró diseñar el Sistema de Actividades físicos- recreativas, quedando conformada en 4 bloques fundamentales: Actividades Gimnásticas en Silla de Ruedas, Actividades Deportivas Adaptadas en Silla de Ruedas, Actividades Recreativas Adaptadas en Silla de Ruedas, Danza Terapia en Silla de Ruedas.

- La validación efectuada muestran la factibilidad, pertinencia y utilidad del Sistema de Actividades físico recreativas, como una solución viable para ser implementada dentro de la asociación Asopléjica de la ciudad de Guayaquil.

\section{Referencias Bibliográficas}

Barroso, G., Cordova, B., \& Calero, S. (2 de 2 de 2016). Metodología para el desarrollo de programas de actividad física adaptada . Parte II diseño y validación. EFdeportes. Recuperado el 4 de agosto de 2018, de http://www.efdeportes.com/efd213/programas-de-actividad-fisica-adaptadaii.htm

Castelo, R Maqueira, G; (2015) "El reconocimiento y desarrollo del esquema corporal en la edad infantil: una experiencia en Ecuador”. EFDeportes.com, Revista Digital. Buenos Aires, año 20, No 209, octubre de 2015. http://www.efedeportes.com/

Herrera, S. (2016). Actividad fìsica recreativa y calidad de vida en adultos mayores. FUNDACIÒN UADE. Recuperado el 3 de febrero de 2019, de https://repositorio.uade.edu.ar/xmlui/bitstream/handle/123456789/4079/Herrera. pdf? sequence $=1 \&$ isAllowed $=\mathrm{y}$

Informe del Consejo Nacional para Discapacidades (2017) Ecuador.

Ladinez, J. (2016). Folklore nacional en las actividades físicas recreativas para elevar el autoestima en los adultos parapléjicos de Asoplèjica de Guayaquil. Guayaquil. $\begin{array}{lllllll}\text { Recuperado } & \text { el } & 1 & \text { de } & 2 & \text { de } & \text { 2019, }\end{array}$ dehttp://repositorio.ug.edu.ec/bitstream/redug/28075/1/Ladinez\%20Garces\%20J ohanna\%20Vanessa\%20035-2016.pdf

Lanuna, T., Laiño , F., \& Castro, H. (junio de 2014). El juego recreativo y el deporte social como polìtica de derecho. Su relaciòn con la infancia en condiciones de vulnerabilidad social . Scielo, 16. doi:2314-2561 
Moreno, M., \& Amaya, M. (2009). Paraplejia pasado y futuro del ser.Scielo.Obtenidodehttp://scielo.isciii.es/scielo.php?script=sci_arttext\&pid=S1 132-12962009000300010

OMS. (23 de FEBRERO de 2018). OMS. Recuperado el 2 de 2 de 2019, de https://www.who.int/es/news-room/fact-sheets/detail/physical-activity

Pérez,D. (23 de 02 de 2017). El deporte como medio de integración e inclusión social. INESEM. Recuperado el 31 de Julio de 2018, de https://revistadigital.inesem.es/educacion-sociedad/inclusion-social-deporte/

Pizarro,G., \& Veintimilla,P. (2016). Atención primaria en discapacidades (1 ed.). Guayaquil: Ciudadano Editorial.

Reina, R. (abril de 2003). Propuesta de intervenciòn para la mejora de actitudes hacia personas con discapacidad a travès de actividades deportivas y recreativas. efdeportes.com. Recuperado el 31 de 01 de 2019 , de https://www.efdeportes.com/efd59/discap.htm

Resumen Informe Mundial la Discapacidad. (2011) Discaapcidad y Rehabilitación.

Rios, M., Arraez, J., Bazalo, P., Enciso , M., Hueli, J., Jimenez, E., Solìs , M. (2016). Plan integral para la actividad fìsica y el deporte (Vol. 1). Recuperado el 2 de 2 de 2019, de http://femede.es/documentos/Discapacidadv1.pdf

Tito,D., Ramiro, M., Rodriguez, A., Analuiza, E., \& Calero, S. (2016). Influencia de las actividades fisico- recreativas en la autoestima del adulto mayor. 35 . Recuperado $\begin{array}{llllll}\text { el } & 1 & \text { de } & 02 & \text { de } & \text { 2019, }\end{array}$ dehttp://scielo.sld.cu/scielo.php?pid=S0864.03002016000400007\&script=sci_art text\&tlng=pt

Sailema A., Maqueira, Sailema, M., \& Sailema, AD., "Estrategia metodológica, esquema corporal y actividad física adaptada: Una trilogía para la inclusión social. Revista Ciencia Digital. ISSN: 2602-8085 Vol. 3, N².5, p. 186-196, abril - junio, 2019.DOI https://doi.org/10.33262/cienciadigital.v3i2.5.53Alonso, G. (2020), Competencias del docente en la atención a estudiantes con conductas disruptivas de 8vo año de Educación General Básica. Tesis en Opción al grado de Magister en Inclusión Educativa y Atención a la Diversidad Universidad Universidad Laica Vicente Rocafuerte. Guayaquil. ( próxima a sustentación)

Campoverde, M. (2014). Desempeño docente en el proceso de enseñanza aprendizaje en el nivel de Educación Básica de la Unidad Educativa "Belisario Quedo" en la ciudad de Pujilí, provincia de Cotopaxi, Año lectivo 2013-2014. Cotopaxi-Ecuador: Universidad Técnico Particular de Loja. 
Cépedes, A. (2012). Niños en Pataletas.adolescentes desafiantes, Como manejar los trastornos de conductas en los hijos.Santiago de Chile: Ediciones Básicas Chile SA

Cuberes, R (2013) Causas de las conductas desafiantes en alumnos de primaria.Trabajo de fin de Grado Universidad Internacional de la Rioja.Facultad de Educación.

Duarte, J. (2017). Formación permanente de docentes en servicio, alternativa para la enseñanza y el aprendizaje de la lengua escrita en la educación básica integral. México: Universidad Rovira.

Freire, C. (2012). Factores que inciden en la presencia de conductas disruptivas de los estudiantes de $\operatorname{los} 8^{\circ}, 9^{\circ} 10^{\circ}$ años en educación básica de la Unidad Educativa experimental FAE N`3 Taura del Cantón Yaguachi durante el período lectivo 2012. Milagro: Universidad Estatal de Milagro.

Flores, G. (2018). Programa de capacitación psicológica a padres de familia para la atención de niños con conductas disruptivas de 6to EGB. Tesis en opción al grado de Magister en Inclusión Educativa y Atención a la Diversidad. Universidad Laica Vicente Rocafuerte. Guayaquil.

Espinoza, G. (2014). Desempeño docente en el proceso de enseñanza aprendzaje en el nivel de Educación Básica Superior del Centro Educativo Colegio de Bachillerato Ciudad de Portovelo. Loja: Universidad Técnica Particular de Loja.

Equipo Dide, 2017, Conductas disruptivas. La asertividad, una competencia docente primordial. http://educaryaprender.es/conductas-disruptivas-comportamiento/

Expasa. (2018). Diccionario Enciclopédico. En T. I.-t. Edición-. Barcelona: Expasa Calpe.

Jiménez, J. (2017). Conductas disruptivas en el Aula y desempeño docentes en la Institución Educativa Técnica General Roberta Leyva del Municipio de Saldaña Tolima. Ibague: Universidad del Tolima.

Jurado, P. (Coord.)(2015), Informe "Influencia de los comportamientos disruptivos en el fracaso escolar de los alumnos de ESO Hacia un modelo de intervención centrado en la institución educativa" Barcelona: UAB (Proyecto I+D-2010. EDU201020105 (subprograma EDUC)

Jurado, P \& Justiano MD(2015), "Las conductas disruptivas y los procesos de intervención en la educación secundaria" Universidad Autónoma de Barcelona (España) Cooperación Técnica Alemana (Bolivia)

Morazán, S. (2013). Competencias docentes y su relación con el rendimiento académico en la asignatura de matemáticas en las instituciones de educación media del municipio de Danlí. Tegucigalpa: Universidad Pedagógica Nacional "Francisco Morazán". 


\section{PARA CITAR EL ARTÍCULO INDEXADO.}

Ladinez Garces, J., \& Maqueira Caraballo, G. de la C. (2020). Actividades físicas-recreativas e inclusión: Una experiencia con adultos parapléjicos de la Asociación Asopléjica. Ciencia Digital, 4(4.1), 32-46. https://doi.org/10.33262/cienciadigital.v4i4.1.1451

\section{Liencia}

El artículo que se publica es de exclusiva responsabilidad de los autores y no necesariamente reflejan el pensamiento de la Revista Ciencia Digital.

El artículo queda en propiedad de la revista y, por tanto, su publicación parcial y/o total en otro medio tiene que ser autorizado por el director de la Revista Ciencia Digital.
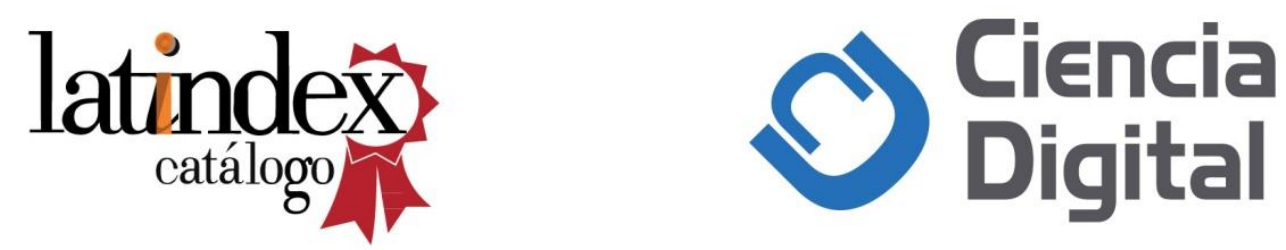